\title{
GROUPS TRANSITIVE ON THE $n$-DIMENSIONAL TORUS
}

\author{
DEANE MONTGOMERY ${ }^{1}$ AND HANS SAMELSON
}

In this note we denote by $G$ a compact connected Lie group. We shall be interested in the situation where $G$ acts as a topological transformation group ${ }^{2}$ on a space $E$. Such a group is called effective if the identity is the only element of $G$ which leaves every point of $E$ fixed. If $G$ is transitive on $E$, that is, for any two points $x$ and $y$ of $E$ there is an element $g$ of $G$ such that $g(x)=y$, then $E$ is called a homogeneous space or a coset space of $G$. Our purpose is to prove the following theorem:

THEOREM. If a compact connected Lie group $G$ is transitive and effective on a space E homeomorphic with an n-dimensional torus (topological product of $n$ circles), then $G$ is isomorphic with the n-dimensional toral group $T_{n}$ (direct product of $n$ circle groups) and no element of $G$ except the identity leaves any point of $E$ fixed.

We use a method of proof which has some similarity to a method we have used in studying groups transitive on spheres. ${ }^{3}$

Let $H^{\prime}$ be a compact, connected, simply connected Lie group, let $T_{l}$ be an $l$-dimensional toral group, and let $N$ be a finite normal subgroup of the direct product $H^{\prime} \times T_{l}$ such that $G$ is continuously isomorphic to the factor-group $\left(H^{\prime} \times T_{l}\right) / N .^{4}$ Let $H^{\prime}$ go into $H$ by the homomorphism obtained by factoring with respect to $N$ and let $T_{l}$ go into $K$. The group $K$ is also an $l$-dimensional toral group, and $H$ and $K$ are subgroups of $G$ which span $G$ or generate $G$. The elements of $H$ commute with the elements of $K$, in fact $K$ is a central subgroup of $G$.

Let $x$ be an arbitrarily chosen point of $E$ and let $H_{x}, K_{x}$, and $G_{x}$ be, respectively, the subgroups of $H, K$, and $G$ which leave $x$ fixed. Let $K^{x}$ be the subgroup of $K$ consisting of those elements $k$ such that $k(x)$ is in the orbit $H(x)$. The orbit $K^{x}(x)$ is the intersection of $H(x)$ and $K(x)$. It can be seen that if $y=g(x)$ then $K_{y}=g K_{x} g^{-1}$ and $H_{y}=g H_{x} g^{-1}$. Since $K$ is a central subgroup we see that $K_{y}=K_{x}$.

Presented to the Society, September 10, 1942; received by the editors September 17,1942

1 Guggenheim fellow.

${ }^{2}$ For the theory of topological groups and Lie groups needed see Pontrjagin, Topological groups, Princeton 1939. For definitions and results concerning topological transformation groups see Zippin, Transformation groups, Lectures in Topology, Ann Arbor 1941 pp. 191-221.

${ }^{3}$ See a paper by us which is forthcoming.

${ }^{4}$ For the existence of these groups see Pontrjagin, loc. cit. pp. 282-285. The group $H^{\prime}$ is the direct product of the simple Lie groups there mentioned. 
Because $G$ is transitive the last remark shows us that every element of $K_{x}$ leaves every point of $E$ fixed, and this together with the fact that $G$ is effective implies that $K_{x}$ contains only the identity element. The orbit $K(x)$ is therefore homeomorphic to $K$ and consequently $l$, the dimension of $K$, must be less than or equal to $n$, the dimension of $E$.

We denote by $\mathfrak{H}_{1}(S)$ the one-dimensional homology group with rational coefficients of the space $S$. If a compact connected Lie group is mapped in the natural way on one of its orbits (namely by considering the orbit as a coset space of the group) then the one-dimensional homology group of the group manifold is mapped onto the onedimensional homology group of the orbit. This follows from the fact that the mapping can be carried out in two steps, the first being a fibering of the group with respect to a connected subgroup, and the second being a finite covering. In both steps the one-dimensional homology groups (with rational coefficients) are mapped onto the one-dimensional homology groups of the respective spaces.

We now apply the above remark to the group $G$ and the orbit $E$. The homology group $\mathcal{H}_{\mathbf{1}}(E)$ is an $n$-dimensional vector space, that is the first Betti number of $E$ is $n$. Therefore the first Betti number of $G$ is at least $n$, and from this we see that the first Betti number of $H^{\prime} \times T_{l}$ must be at least $n$. However the first Betti number of $H^{\prime} \times T_{l}$ is $l$ and we see that $l$ is greater than or equal to $n$.

The above results show that $l$ equals $n$. It follows that the orbit $K(x)$ is the whole of $E$ which means that $K$ is transitive on $E$. We see therefore that $K^{x}(x)=H(x)$. Since $K^{x}(x)$ is homeomorphic to $K^{x}$ and since $H(x)$ is connected it follows that $K^{x}$ is connected. Therefore $K^{x}$ as a connected subgroup of a toral group must itself be a toral group of some dimension greater than zero or it must contain only, the identity element. But from the fact that $\mathfrak{H}_{1}(H)=0$ it follows that $\mathfrak{F}_{1}[H(x)]=0$. Hence $\mathcal{F}_{1}\left(K^{x}\right)=0$ and $K^{x}$ contains only the identity element, and $H(x)=x$. The point $x$ was chosen arbitrarily so that the equation $H(x)=x$ holds for every $x$ in $E$. Because $G$ is effective this means that $H$ contains only the identity element and that $G=K$ which proves the theorem.

The same proof applies if instead of assuming that $E$ is an $n$-dimensional torus we merely assume that it is an $n$-dimensional space the first Betti number of which is $n$. It then follows in view of the above proof that $E$ is a torus. If we drop the assumption that $G$ is effective the "effective group" will be a toral group of dimension $n$ as before.

Smith College and

The Institute for Advanced Study 